\title{
Twin to twin transfusion syndrome
}

\section{Bhat R}

Associate Professor, Department of Paediatrics, Kasturba Medical College, Manipal University, Manipal, Karnataka, India

\begin{abstract}
Twin to twin transfusion syndrome (TTTS) is a specific condition complicating $10-15 \%$ of monochorionic multiple pregnancy. Intertwin vascular anastomoses and transfusion is a normal event. Syndrome develops when blood flow is unbalanced. Recent studies suggest a paucity of bi-directional superficial anastomoses failing to compensate for hemodynamic imbalance resulting from unidirectional transfusion along deeper arterio-venous anastomoses. TTTS is associated with high rates of perinatal mortality. Acute and chronic forms exist and severity varies. The donor twin is often smaller, hypovolemic, anemic and develops congestive heart failure. The recipient is usually heavier and polycythemic. Antenatally, second trimester ultrasound usually detects the condition. Serial amnioreduction is the recommended therapy. Postnatally, intertwin haemoglobin and birth weight differences along with pallor in one twin and plethora in the other are helpful in making a diagnosis. Medical care of twins after birth is directed towards problem related to anemia, polycythemia and hydrops. Review of the syndrome is presented with an illustrative case.
\end{abstract}

Key words: Twin to twin transfusion syndrome, Neonatal diagnosis, Symptomatic polycythemia, Partial exchange transfusion

$\mathrm{T}$ win to twin transfusion syndrome (TTTS) is a specific complication of monozygotic twins with monochorionic placentation resulting from transfusion of blood from one twin to the other through a deep, artery to vein placental vascular anastomosis. Consequently, the donor twin becomes anaemic, hypovolaemic, hypotensive, and hypoproteinaemic, undergoes intrauterine growth retardation, and occasionally develops oligohydramnios. In contrast, the recipient twin is heavier, polycythaemic, hypervolaemic, and faces complications of hyperviscosity of the blood such as cardiac failure, hyperbilirubinaemia, and intravascular thrombosis. The degree of severity of the twin transfusion is dependent on the duration of pregnancy, structural alterations of the vasculature and chronicity of transfusion. Neonates are considered having TTTS if any two of the following criteria are satisfied: 1) intertwin haemoglobin difference $>5 \mathrm{~g} / 100 \mathrm{ml}(\mathrm{PCV}>15 \%)$, (2) pallor in one twin and plethora in the other, (3) inter-twin birth weight difference $>15 \%{ }^{1,2}$. In acute form birth weight discrepancy may be less than $15 \%$ and haemodynamic problems such as tachycardia or hypotension may be present.

\section{Case Report}

A pair of twins was born to 37 -year-old $2^{\text {nd }}$ gravida mother through vaginal route at term gestation. The first was born as vertex and the second as breech at an interval of five minutes. Both were male with B positive blood groups. Placenta was monochorionic and diamniotic. The first was smaller with birth weight of $2300 \mathrm{~g}$, pale with PCV of $39 \%(\mathrm{Hb}-13 \mathrm{~g} / \mathrm{dl})$. The second was heavier with birth weight of $2650 \mathrm{~g}$, plethoric and (Hb-23g/dl) PCV of $69 \%$ (Fig1A). First was pale but asymptomatic. The second had symptomatic polycythemia with plethora, lethargy, hyperbilirubinemia, hypoglycemia and elevated serum creatinine. Peripheral smear examination showed normocytic, normochromic RBCs in both twins, decreased RBC mass in the first and increased $\mathrm{RBC}$ mass in the second twin. Diagnosis of twin to twin transfusion was considered in this monochorionic twins ${ }^{2}$. Partial exchange transfusion was carried out for polycythemic, symptomatic $2^{\text {nd }}$ twin. Post exchange this twin was less plethoric (Fig $1 \mathrm{~B})$. His cranial and renal ultrasounds were normal. Subsequently he became asymptomatic and started breast feeding well. Both were discharged after a week. The antenatal ultrasound was reported as normal. At six months follow up both the twins were normal in terms of growth and development. Though $2^{\text {nd }}$ trimester

Correspondence

Dr. Ramesh Bhat

Associate Professor

Department of Paediatrics, Kasturba Medical College

Manipal University, Manipal

Karnataka 576104, India

E-mail: docrameshbhat@yahoo.co.in 
ultrasound detects this condition, at many instances diagnosis is still made after birth.

\section{Frequency of TTTS}

Approximately $75 \%$ of monozygotic twins are monochorionic. TTTS occurs in 5-38\% of monochorionic twins. The extreme variant of TTTS, the "stuck twin" phenomenon has been reported in $8 \%$ of twin pregnancies. Acute severe TTTS occurs in 1\% of monochorionic twin pregnancies. It may present as acute hydramnios resulting in preterm labour or premature rupture of membranes $\mathrm{s}^{3,4,5}$.

\section{Pathophysiology}

Monozygotic twins with monochorionic, diamniotic placentation or monochorionic, monoamniotic placentation are at risk. TTTS results from transfusion of blood from one fetal twin to another twin through placental vascular anastomoses commonly in a deep, artery-to-vein anastomosis through a shared placental cotyledon. When the cotyledon is supplied by an artery from one twin and drained by a vein from the other twin, there is potential for transfusion from the donor twin (on the arterial side) to the recipient twin (on the venous side). Consequently, the donor twin becomes anaemic, hypovolaemic, hypotensive, and hypoproteinaemic, undergoes intrauterine growth retardation, and occasionally develops oligohydramnios. In contrast, the recipient twin is heavier, polycythaemic, hypervolaemic, and faces complications of hyperviscosity of the blood such as cardiac failure, hyperbilirubinaemia, and intravascular thrombosis.

A

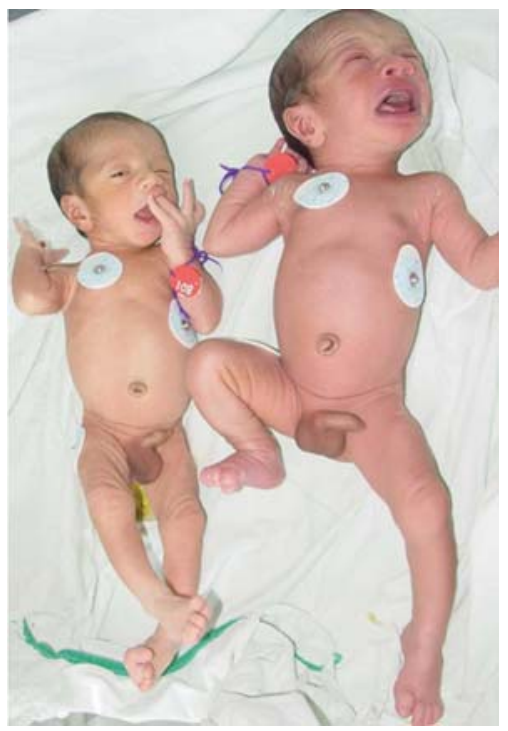

Fig 1 A: Twin to twin transfusion syndrome, the smaller pale donor and heavier, plethoric recipient
The degree of severity of the twin transfusion is dependent on the duration of pregnancy, structural alterations of the vasculature (vessel caliber, quality of the anastomoses, and the presence/absence of vascular communication in the opposite direction to compensate for the haemodynamic imbalance), and chronicity of transfusion. Severe oligohydramnios can result in the stuck twin phenomenon in which the twin appears in a fixed position against the uterine wall ${ }^{3-6}$.

When the birth weight discrepancy between twins is small, the transfusion is considered to have occurred acutely. A distinction between the acute and chronic forms of the syndrome can be made on the basis of weight discrepancy and haemodynamic changes. Infants with the chronic form have discrepancies in birth weight exceeding $15 \%$ and the peripheral blood film of the donor twin may show hypochromic microcytic anaemia and erythroblastosis. The acute forms may be one of the emergencies encountered in the delivery room. Severe anaemia and shock may occur in the donor and require urgent transfusion of blood. The recipient twin may be in cardiac failure and require urgent reduction of his/her blood volume by withdrawal of blood from the umbilical vein. Cord haemoglobin may be misleading in the acute forms of the syndrome, as they may be normal with no intrapair discrepancy ${ }^{2,6}$. In such instances, a full blood count performed between six and 12 hours after delivery will show the characteristic intrapair discrepancy. A high mortality ranging between 79 and $100 \%$ has been reported for twins with the acute types presenting at $18-26$ weeks.

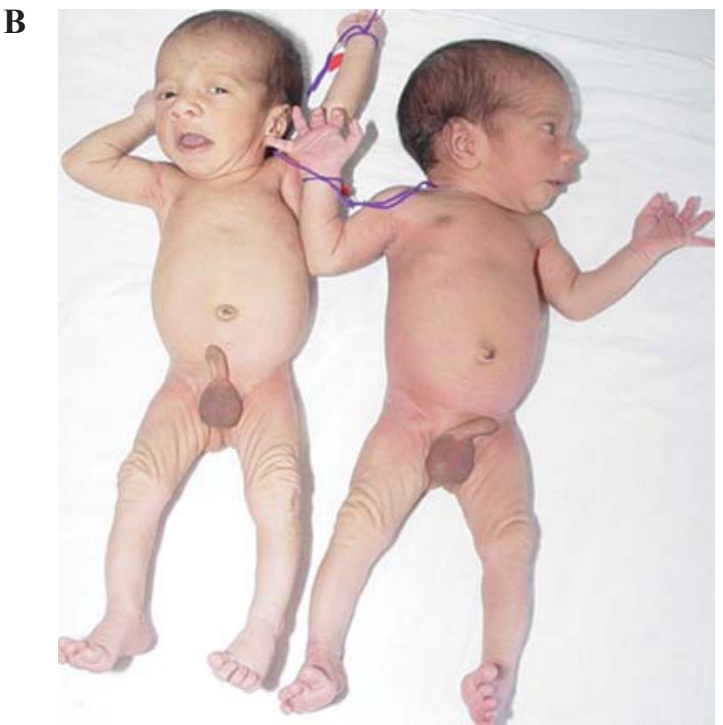

B: Less plethoric recipient after partial exchange transfusion 


\section{Diagnosis}

Antenatal: Pregnant woman with multiple pregnancies who have TTTS may frequently complain of a rapidly enlarging abdomen. There may be preterm labor and premature rupture of membranes. Physical examination may reveal rapidly increasing fundal height. The diagnosis of twin-twin transfusion syndrome is largely based on sonographic findings of disparate liquor volumes in the two amniotic sacs ${ }^{7}$. Additional findings may include a stuck donor with nonvisualization of the bladder, arterial and/or venous doppler abnormalities, hydrops, and an absence of arterioarterial anastomoses.

Neonatal: Following birth, TTTS is considered in monochorionic twins if any two of the following criteria are satisfied: 1) intertwin haemoglobin difference $>5$ $\mathrm{g} / 100 \mathrm{ml}, 2$ ) pallor in one twin and plethora in the other, 3 ) intertwin birthweight difference $>15 \%$. In acute form of TTTS birth weight discrepancy may be less than $15 \%$ and haemodynamic problems such as tachycardia or hypotension may be present. Donor twin is usually small for gestational age, more than $20 \%$ smaller than recipient twin, pale with poor peripheral perfusion. The recipient twin is large for gestational age, more than 20\% larger than donor twin, plethoric, ruddy and jaundiced. Hydrops fetalis can be present in either twin These infants have subcutaneous edema, a distended abdomen, and respiratory distress.

\section{Spectrum of TTTS}

Spectrum of the syndrome varies from mild to severe. Severe TTTS manifests at 16-18 weeks gestation. Hemoglobin concentrations are usually the same in both twins. Polyhydramnios develops in the sac of the recipient twin and oligohydramnios in the sac of the donor twin. The stuck twin phenomenon may appear. Moderate TTTS develops sometime between 24-30 weeks' gestation. Polyhydramnios and oligohydramnios do not develop. The donor twin becomes anemic and hypovolemic, and growth is retarded. The recipient twin becomes plethoric, hypervolemic and macrosomic. Either twin can develop hydrops fetalis. The recipient twin can also develop hypertension, hypertrophic cardiomegaly, disseminated intravascular coagulation, and hyperbilirubinemia after birth. Mild TTTS develops slowly in the third trimester. Polyhydramnios and oligohydramnios usually do not develop. Hemoglobin concentrations differ by more than $5 \mathrm{~g} / \mathrm{dl}$. Twin size differs by more than $20 \%$. Acute TTTS can occur at birth during the time between clamping the umbilical cords of the first and second twin.

\section{Morbidity/Mortality}

Severe TTTS has a $60-100 \%$ mortality rate. Mildto-moderate TTTS is frequently associated with premature delivery. Morbidity and mortality depend upon the gestational age of the twins at delivery. The more premature the twins are at birth, the higher the incidence of morbidity and mortality. Fetal demise of one twin is associated with neurologic sequelae in $25 \%$ of surviving twins. A high mortality ranging between 79 and $100 \%$ has been reported for twins with the acute types presenting at 18-26 weeks. Intrauterine demise of one twin can result in neurologic sequelae in the surviving twin. Acute exsanguination of the surviving twin into the relaxed circulation of the deceased twin can result in intrauterine CNS ischemia.

Differential diagnosis include hydrops fetalis, polycythemia, polyhydramnios, oigohydramnios and anaemia of the newborn

\section{Laboratory Studies}

Complete blood count (CBC) suggests the anaemia and polycythemia. Hypocalcemia is frequently present in the donor twin. Hypoglycemia may be present in either twin. Either twin may have evidence of renal dysfunction and raised creatinine. Thrombocytopenia can occur in either twin. Hyperbilirubinemia may develop in the polycythemic recipient twin.

\section{Imaging Studies}

Head ultrasonography is helpful in detecting intraventricular hemorrhage and periventricular leukomalacia. Myocardial dysfunction, myocardial hypertrophy, valvular insufficiency, and pericardial effusions can be detected in either twin by echocardiography. Renal ultrasonography may detect abnormalrenalechogenicityindicatinghypoxic-ischemic cortical necrosis that may be present in either twin. If hydrops fetalis occurs, abdominal ultrasonography may detect ascites and chest radiography is helpful for identifying pleural effusions and cardiomegaly

\section{Medical Care of affected twins}

Antenatal therapeutic options include laser photocoagulation of chorionic plate vessels, serial amnioreduction, septostomy, and selective feticide. Selective fetoscopic laser photocoagulation of chorioangiopagus has emerged as an alternative treatment strategy in utero therapeutic intervention. Amnioreduction and septostomy are simple procedures, which primarily control amniotic fluid volume but may also ameliorate other features of the disease ${ }^{8-11}$. In contrast, laser ablation and selective feticide are technically difficult procedures. A recent meta-analysis has shown that the overall survival rates for twintwin transfusion syndrome cases presenting at less than 28 weeks treated by laser or amnioreduction are similar ${ }^{11,12,13}$. 
Medical care of twins after birth is directed toward problems related to prematurity, anemia, polycythemia, and hydrops fetalis. Severely anemic donor twins may require packed RBC transfusions or partial exchange transfusions. Polycythemic recipient twins may require partial exchange transfusion to lower serum hematocrit levels. Newborns with hydrops fetalis may require mechanical ventilation, thoracocentesis, pericardiocentesis, and paracentesis. Prompt recognition and management of the haemodynamic and haematological problems of infants with the TTTS results in optimal neurodevelopmental outcome ${ }^{2,14}$.

\section{Prognosis}

Outcome is dependent upon gestational age at birth. The lower the gestational age at birth the greater the risk for long-standing neurologic or pulmonary sequelae. In post-natally diagnosed twins, delay in recognising and treating the neonatal problems may also contribute to long term morbidity. Catch-up growth occurs postnatally in most of the smaller donor twins ${ }^{5,14}$.

\section{References}

1. Lopriore E, Vandenbussche FP, Tiersma ES, et al. Twin-to-twin transfusion syndrome: new perspectives. J Pediatr. 1995;127:675-80.

2. Seng YC and Rajadurai V S. Twin-twin transfusion syndrome: a five year review Arch Dis Child. Fetal Neonatal Ed. 2000;83:168-70.

3. Keith RD, Mark LD, Nicholas M F. Prenatal diagnosis. 1997; 17:1227-36.

4. Cincotta RB, Fisk NM. Current thoughts on twin-twin transfusion syndrome. Clin Obstet Gynecol. 1997;40:290-302.

5. Berghella V, Kaufmann M. Natural history of twin-twin transfusion syndrome. J Reprod Med. 2001;46:480-4
6. Fox C, Kilby MD, Khan KS. Contemporary treatments for twin-twin transfusion syndrome. Obstet Gynecol. 2005;105:1469-77.

7. Wittmann BK, Baldwin VJ, Nichol B. Antenatal diagnosis of twin transfusion syndrome by ultrasound. Obstet Gynecol. 1981;58:123.

8. Crombleholme TM. The treatment of twintwin transfusion syndrome. Semin Pediatr Surg. 2003;12:175-81.

9. Machin GA, Keith LG. Can twin-to-twin transfusion syndrome be explained, and how is it treated? Clin Obstet Gynecol. 1998;41:10413.

10. Milner R, Crombleholme TM. Troubles with twins: fetoscopic therapy. Semin Perinatol. 1999;23:474-83.

11. Lopriore E, Sueters M, Middeldorp JM et al. Neonatal outcome in twin-to-twin transfusion syndrome treated with fetoscopic laser occlusion of vascular anastomoses. J Pediatr. 2005; 147:597-602.

12. Mari G, Detti L, Oz U, Abuhamad AZ. Longterm outcome in twin-twin transfusion syndrome treated with serial aggressive amnioreduction. Am J Obstet Gynecol. 2000; 183:211-7.

13. Hubinont $\mathrm{C}$, Bernard $\mathrm{P}$, Mwebesa $\mathrm{W}$ et al. Nd: YAG laser and needle disruption of the interfetal septum: A possible therapy in severe twin-totwin transfusion syndrome. J Gynecol Surg. 1996;12:183-97.

14. McCulloch K. Neonatal problems in twins. Clin Perinatol. 1988; 15:141-54. 